\title{
Un peu de lumière sur les neurones autofluorescents: isolement du gène de la maladie de Batten
}

Parmi les maladies neurodégénératives de l'enfant, les lipofuscinoses céroïdes (neuronal ceroid lipofuscinosis, NCL) forment un groupe bien défini, tant du point de vue clinique que biologique. Elles se manifestent par une encéphalopathie précoce, avec perte progressive de la vision et crises d'épilepsie, puis apparaissent des troubles de la motricité et une détérioration mentale irréversible. Parmi les critères d'inclusion diagnostiques, la mise en évidence de lipopigments autofluorescents (lipofuscine et céroïde) dans les neurones et dans d'autres cellules est déterminante [1]. Ces substances accumulées en inclusions cytosoliques donnent, au microscope électronique, des images à type d'empreintes digitales ou de stries linéaires contournées caractéristiques [2]. Le mode de transmission des NCL est récessif autosomique. Leur fréquence est évaluée aux alentours de 1/25000 naissances, mais elles sont beaucoup plus répandues dans les populations de l'Europe du Nord et certaines formes n'ont été décrites qu'en Finlande.

Cing types au moins de NLC peuvent être dénombrés, la classification reposant surtout sur l'âge d'apparition des premiers signes. La forme infantile la plus précoce, la NLC1 (maladie d'Haltia-Santavuori) débute vers l'âge d'un an. Le gène, encore inconnu, est localisé sur le chromosome 1. La NLC2 ou maladie de JanskyBielschowsky a un début plus tardif (3 ans). Quant à la NLC3 (maladie de Batten ou maladie de SpielmeyerVogt) dite juvénile, elle débute vers l'âge de 8 ans. C'est la plus commune et désormais la mieux connue des NLC puisque le gène en cause vient
L'histoire des différentes étapes ayant abouti à cette découverte est exemplaire. Dès 1989 [4], on savait que le gène responsable de la NLC3 était situé sur le chromosome 16 en raison d'une liaison génétique avec l'haptoglobine (dont le gène est localisé sur le bras long du 16). Puis, à l'aide de marqueurs polymorphiques, une équipe parvint à situer le locus dans une région encore imprécise, au-dessous ou au-dessus du centromère [5]. Grâce à des microsatellites, cette même équipe réussit à resserrer la région du locus, en 16p12.1, entre les marqueurs D16S148 et D16S150 [6]. C'est alors qu'une particularité attira l'attention: les malades étaient toujours porteurs des mêmes allèles pour un certain nombre de microsatellites proches du locus. Une étude élargie fut donc entreprise avec de nombreux malades appartenant à 70 familles dont les origines ne sont pas uniquement nordiques. Un net déséquilibre de liaison fut confirmé et un haplotype identique, avec les mêmes allèles pour les microsatellites $D 16 S 298$ et D16S299, fut retrouvé dans $73 \%$ des chromosomes des sujets atteints [7]. Cet haplotype, baptisé « 56 ", aida à situer le gène dans une région de $8 \mathrm{cM}$ et suggéra fortement un effet fondateur. Une équipe hollandaise, ayant rassemblé, les malades des Pays-Bas, décida d'étudier un enfant marocain issu d'une union consanguine afin de savoir si, hypothèse peu probable, il serait, lui aussi, porteur du même haplotype que les malades du nord de l'Europe. Il n'en était rien et on trouva chez lui, à l'état homozygote, une délétion ayant emporté le microsatellite D16S298. Comme on pouvait s'y attendre du fait de leur apparentement, ses parents étaient tous deux porteurs de cette même délétion à l'état hétérozygote.

Étant donc près du but, et pour plus d'efficacité, les équipes ayant obtenu ces résultats se regroupèrent alors dans le "Consortium international de la maladie de Batten ". Le cosmide contenant D16S298 et englobant la délétion de l'enfant marocain avait toutes les chances de contenir aussi le gène. A l'aide de ce cosmide, par amplification d'exons et criblage d'une banque d'ADNc provenant de cerveau fœetal, un gène candidat fut trouvé et son rôle dans la maladie de Batten démontré puisqu'on observe des mutations dans ce gène chez tous les malades. Ceux qui étaient porteurs de l'haplotype 56 ont, évidemment, la même lésion originelle: il s'agit d'une délétion de $1,02 \mathrm{~kb}$; elle entraîne une rupture dans le cadre de lecture avec pour conséquence une protéine tronquée dans sa partie C-terminale; $81 \%$ des malades étudiés jusqu'à présent ont l'haplotype 56 mais, outre les délétions, des mutations ponctuelles sont observées chez quelques patients. La confirmation définitive de la responsabilité du gène $C L N 3$ dans la maladie de Batten serait apportée si l'invalidation du gène chez l'animal entraînait les manifestations de la maladie de Batten. On peut noter que le gène est conservé chez les mammifères.

Il code pour une protéine de 438 acides aminés dont la structure ne semble présenter aucune similitude avec celle des protéines déjà connues. Sa structure primaire indique une protéine membranaire de type IIIb, avec de nombreux sites potentiels de glycosylation, de myris- 
toylation et de phosphorylation. On ne peut exclure la possibilité, chez certains malades, de la présence d'un gène $C L N 3$ normal mais d'anomalies de sa régulation fonctionnelle.

La perte de fonction de la protéine semble responsable de la maladie de Batten, comme le suggère la survenue de la maladie chez des sujets présentant une délétion totale du gène. Mais on ne sait pas si, dans les cas comportant des délétions partielles, et en particulier l'haplotype 56, la protéine tronquée est synthétisée et s'accumule dans divers tissus. On n'a, toutefois, pas encore établi de relation entre le phénotype clinique et l'anomalie génétique. L'élément essentiel de la substance accumulée dans les inclusions cytosoliques est la sous-unité 9 de l'ATPase mitochondriale, dont les gènes, connus, ne sont pas en cause. De même, plusieurs voies métaboliques sont altérées, dont la peroxydation lipidique, le métabolisme des oligosaccharides liés au dolichol, l'activité protéasique des lysosomes. Il devient donc urgent et important de comprendre le mécanisme pathogénique de cette maladie pour pouvoir envisager ultérieurement un traitement spécifique. De plus, elle pourrait bien servir de modèle à d'autres neurodégénérescences et être, de ce fait, très éclairante en neurogénétique et dans certaines maladies de surcharge. Mais dès maintenant la connaissance du gène et de ses mutations permet d'étayer le diagnostic clinique (qui n'est pas facile, surtout au début de la maladie), et d'aider les familles en rendant accessible le diagnostic prénatal et le dépistage des hétérozygotes.

S.G.
1. Dyken PR. Reconsideration of the classification of the neuronal ceroid lipofuscinosis. Am J Med Genet 1988; 5 (suppl) : 69-84.

2. Wisniewski KE, Rapin I, Heany-Kieras J. Clinicopathological variability in the childhood neuronal ceroid lipof uscinoses and new observations on glycoprotein abnormalities. Am J Med Genet 1988; 5: $27-46$

3. The international Batten disease consortium Isolation of a novel gene underlying Batten disease, CLN3. Cell 1995; 82: 949-57.

4. Eiberg H, Gardiner RM, Mohr J. Batten disease (Speilmeyer-Sjogren disease) and haptoglobin (HP): indication of linkage and assignment to chromosome 16. Clin Genet 1989; 36: 217-8.

5. Gardiner RM, Sandford A, Deadman M, Poulton J, Cookson W, Reeders S, Jodiako l, Peltonen L, Eiberg H, Julier C. Batten disease (SpielmeyerVogt disease, juvenile onset neuronal ceroid lipofuscinose) gene (CLN3) maps to human chromosome 16. Genomics 1990; 8: 387-90.

6. Yan W, Boustany RMN, Konradi C, Ozelius L, Lerner T, Trof atter JA, Julier C, et al. Localization of juvenile, but not late-infantile, neuronal ceroid lipofuscinosis on chromosome 16. Am J Hum Genet 1993; 52: 89-95.

7. Lerner TJ, Boustany RMN, Mac Cormack K, Gleitsman J, Schlumpf K, Gusella JF, Breakefield $\mathrm{XO}$, Haines JL. Linkage desequilibrium between the juvenile NCL gene (NCL3) and marker loci on chromosome 16p12.1. Am J Hum Genet 1994; 54: 88-94.
Le jus de carotte comme traitement d'une maladie génétique? Parmi les dégénérescences rétiniennes d'origine génétique, une forme rare, la dystrophie du fond d'œil de Sorsby (SFD), est la seule qui comporte des lésions maculaires hémorragiques comme on en observe dans la dégénérescence maculaire liée à l'âge (ARMD), principale cause de cécité chez le sujet âgé dans le monde occidental. JeanClaude Dreyfus avait récemment passé en revue les dystrophies maculaires de la rétine et expliqué le mécanisme par lequel les mutations du gène TIMP3 entraînent un changement dans l'action de la métalloprotéinase 3 , normalement soumise au contrôle de cet inhibiteur chez les malades, ainsi que la présence d'un dépôt jaunâtre dans la membrane de Bruch* constituant une gêne à la vascularisation $\left(m / s n^{\circ} 2\right.$, vol. 11, p. 295). Il était logique de supposer que le trouble de la vision nocturne et les lésions du fond d'œil observés dans la dystrophie de Sorsby seraient la conséquence d'un apport insuffisant de vitamine A aux photorécepteurs, en raison de l'atteinte capillaire. C'est ce que vient de démontrer un groupe américain [1]. Dans une famille atteinte de SFD sur quatre générations, la mutation trouvée est une substitution d'un nucléotide au codon 167 , créant un nouveau site de restriction pour l'enzyme Bbvl, ce qui rend facile la détection des sujets atteints. L'administration de vitamine A (50 $000 \mathrm{UI} /$ jour) fait spectaculairement disparaître la cécité nocturne chez les malades et pourrait être proposée, de façon prophylactique, aux sujets plus jeunes n'ayant pas encore de manifestations cliniques (en tenant compte toutefois de la toxicité potentielle de la vitamine A pour l'ajustement des doses). Malheureusement, malgré sa richesse en cette vitamine et en contradiction avec le titre de cette nouvelle, il n'est pas sûr que la carotte soit suffisamment riche en carotène pour remplacer les préparations de vitamine A purifiée! Enfin, et même si certains mécanismes physiopathologiques semblent analogues, il n'y a que peu de chances que la vitamine A puisse avoir une réelle efficacité sur la dégénérescence maculaire liée à l'âge, en raison de sa diversité étiologique.

[1. Jacobson SG, et al. Nature Genet $1995 ; 11: 27-32$.]

* Mince lame de tissu élastique et de fibres de collagène interposée entre l'épithélium pigmentaire de la rétine et la choroïde. 
Comment se comporte la séquence instable de l'X fragile dans un tissu tumoral? Si la question valait d'être posée, on va voir que les résultats, fort intéressants, méritent réflexion. Chez un sujet atteint de maladie de l' $\mathrm{X}$ fragile et décédé d'un cancer bronchique, l'étude de l'expansion de la séquence CGG, de sa méthylation et de l'expression du gène $F M R 1$ a été réalisée à partir de l'ADN de différents tissus, y compris de la tumeur [1]. On sait que, dans la maladie de l'X fragile, la présence d'une mutation complète, avec expansion des répétitions CGG aux alentours de 200 ou plus, s'accompagne d'une hyperméthylation, responsable de l'absence de synthèse de la protéine FRMP. On sait aussi que les mosaïques sont très fréquentes chez les malades: des cellules porteuses d'une prémutation (sans méthylation et donc avec expression de FMRP) accompagnent, parfois en petit nombre, les cellules ayant la mutation complète. De plus, l'expérience a montré que la lignée germinale des sujets “X fragile" ne porte jamais la mutation complète mais seulement une prémutation. Il faut donc tenir compte de toutes ces données pour interpréter les résultats observés dans les ADN du malade étudié. Son phénotype était caractéristique de la maladie de l'X fragile et la plupart des tissus, étudiés en Southern blot, montraient une mutation complète avec méthylation. Dans le tissu testiculaire, on trouve la mutation complète ainsi qu'une prémutation qui correspond, comme on pouvait s'y attendre, à la lignée germinale. Quelques tissus révèlent, en outre, l'existence d'une mosaïque (mutation complète et prémutation dont l'expansion est différente de celle de la lignée germinale) confirmée par l'observation en immunohistochimie des coupes de tissu cérébral, $1 \%$ des neurones du cortex exprimant $F M R P$. Dans la tumeur, FMRP est présente dans $30 \%$ à $40 \%$ des cel- lules, sans mutation complète mais avec seulement une prémutation. La tumeur doit donc provenir d'une des rares cellules somatiques préexistantes avec prémutation. Mais, fait surprenant, la région prémutée est cependant méthylée, y compris les îlots $\mathrm{CpG}$. Dans ces conditions, comment FMRP a-t-il pu être exprimé et existe-t-il des mécanismes de régulation encore inconnus? De telles observations démontrent une fois de plus la labilité de la séquence instable $\left(\mathrm{m} / \mathrm{s} n^{\circ} 6-7\right.$, vol. 10, p. 747) et permettent d'explorer le fonctionnement du gène FMR1 dans différentes conditions in vivo.

[1. De Graaf E, et al. Am J Hum Genet 1995 ; 57 : 609-18.]

Oreille pâle ou œil rubis ? Un albinisme oculo-cutané particulier. Le syndrome d'Hermansky-Pudlak (HPS) est une maladie assez étonnante, le trouble de la pigmentation étant associé à des anomalies de la fonction plaquettaire et surtout à l'accumulation d'une substance fluorescente composée de lipofuscine et de céroïde dans les cellules endothéliales. De transmission récessive autosomique, cette maladie de surcharge peut être extrêmement sévère avec fibrose pulmonaire et colite granulomateuse. Elle est fréquente dans la population portoricaine $(1 / 1800)$, et une équipe de Minneapolis est partie de l'hypothèse d'une homogénéité génétique, avec un effet fondateur dans l'île de Porto Rico [1]. Comme un certain nombre de gènes intervenant dans la pigmentation sont déjà localisés chez la souris, les analyses de liaison ont porté prioritairement sur les chromosomes humains correspondant aux chromosomes de souris portant ces loci. Une localisation a été trouvée assez rapidement sur le chromosome 10 en 10q23. Dans toutes les familles étudiées, le gène est situé dans une région de $0,6 \mathrm{cM}$ entre les marqueurs polymorphiques D10S577 et D10S198 [2]. Un net déséquilibre de liaison est en outre observé. Dans un isolat suisse, on retrouve la même localisation ainsi qu'un déséquilibre de liaison [3]. Mais la forme suisse est beaucoup moins sévère, l'espérance de vie n'est pas compromise comme dans les cas portoricains et il n'existe aucun accumulation de cérofuscine dans les cellules endothéliales. On peut invoquer une hétérogénéité allélique mais tant que le gène ne sera pas isolé, il serait prématuré de conclure car, chez la souris, il n'y a pas moins de treize gènes dont les mutations entraînent des troubles analogues à cette maladie [3]. Parmi ceux-ci, deux mutations, ruby eye (ru) et pale ears (ep) étroitement liées sont localisées dans une région du chromosome 19 de la souris, correspondant à la région $10 \mathrm{q} 23 \mathrm{chez}$ l'homme. L'exploration de cette région $10 \mathrm{q} 23$ à la recherche d'un ou de plusieurs gènes candidats vaut donc la peine d'être entreprise. Une fois le gène humain connu, il serait facile d'étudier le gène murin analogue. Mélanosomes, thrombocytes et lysosomes n'ayant pas d'origine commune, le gène de HPS touche peut-être un organite intra-cellulaire, ce qui ne manque pas d'intérêt. Beaucoup reste à comprendre dans les troubles de la pigmentation.

[1. Wildenberg SC, et al. Am J Hum Genet 1995 ; 57: 755-65.]

[2. Fukai K, et al. Hum Mol Genet $1995 ; 4$ : 1665-70.]

[3. Schallreuter KU, et al. Dermatology 1993 ; 187 : 248-56.]

Fréquence du gène de l'hémochromatose. L'hémochromatose est une maladie génétique autosomique récessive, dont le gène est lié au locus HLA, et qui a été considérée comme rare. Des études épidémiologiques limitées sont habituel- 


\section{BRÈVES}

lement parties de la prévalence des homozygotes pour conclure à une fréquence génique de 0,02 à 0,10 . Une étude génétique préalable, portant sur 232 malades hémochromatosiques et leurs familles, avait permis l'évaluation de la saturation de la transferrine chez les sujets normaux, hétérozygotes et homozygotes pour l'hémochromatose. Un travail récent, reposant sur cette hypothèse que l'état hétérozygote affecte la distribution de la saturation de la transferrine, a utilisé les données d'une enquête américaine (NHANES II, pour Second National Health and Nutrition Examination Survey) portant sur 21000 sujets de race blanche pour évaluer les proportions respectives de sujets hétérozygotes et non affectés [1]. L'analyse statistique divise clairement deux groupes : $85 \%$ de la population peut être considérée comme homozygote normale, $15 \%$ comme hétérozygote pour l'hémochromatose. Cette fréquence génique de 0,08 coexiste avec une légère prédominance masculine ( $15 \%$ contre $13 \%$ ) ; les valeurs observées dans les deux groupes, normaux et hétérozygotes, se chevauchent très peu (28 ou $29 \% \pm 3 \%$ contre 47 ou $45 \% \pm$ $8 \%)$. L'état hétérozygote étant en lui-même dénué de manifestations pathologiques, il n'est pas question de suggérer une exploration systématique. Des valeurs anormales de la saturation de la transferrine doivent cependant constituer un signe d'appel quand elles coexistent avec certains états hémolytiques chroniques, tels qu'une anémie sidéroblastique, une sphérocytose héréditaire, un déficit en pyruvate kinase par exemple. On a aussi impliqué le gène de l'hémochromatose dans les manif estations d'une porphyrie cutanée tardive. Un diagnostic précoce et un traitement approprié peuvent alors prévenir efficacement les manifestations pathologiques d'une surcharge en fer.

[1. McLaren CE, et al. Blood 1995; 86: 2021-7.]
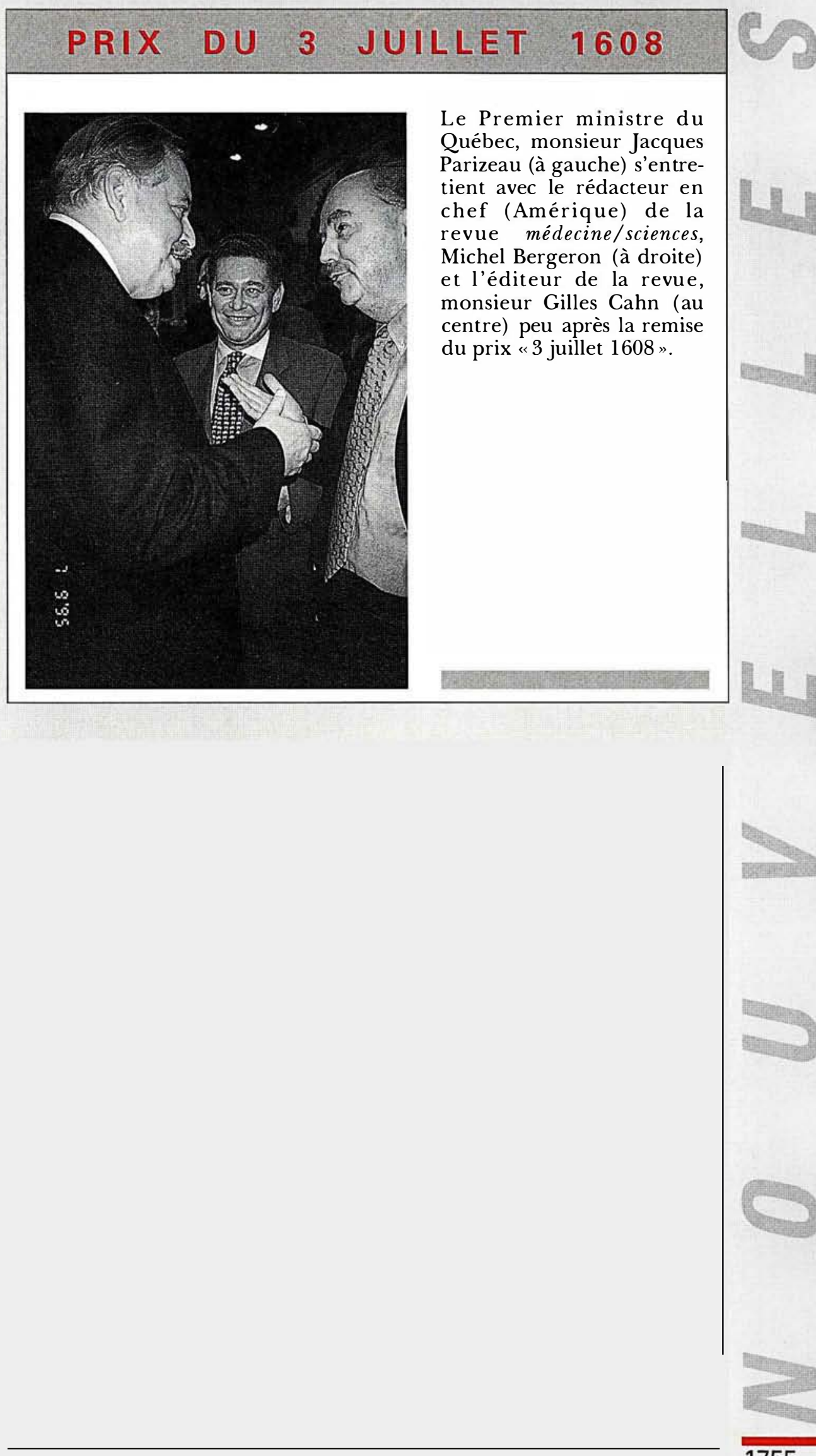